\title{
The past, present and future of sleep medicine
}

\author{
M. S. Kanwar \\ Sr. Consultant Respiratory Medicine, Critical Care \& Sleep Medicine. Founder Apollo Sleep Disorder Institute

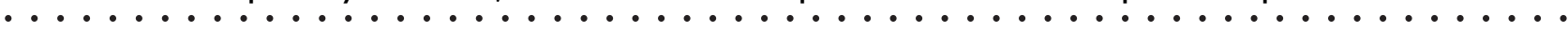 \\ Indian J Sleep Med 2009; 4.4, 149-153
}

\section{The past}

S leep has always been a fascinating one-third of century ago. Since the middle of twentieth century it has been established that sleep is a special activity of brain, controlled by elaborate and precise mechanisms. Before that, circardian behaviour of sleep was theorized in 1922. Sleep was supposed to be the "turning off" of the brain on the human electroencephalogram (EEG) which was getting established in 1930s. Used much in the same way today the recognition of EEG waveforms was a turning point in the sleep research. Researchers favoured the concept of sleep as a reduction of activity - idling, slow, synchronized, resting neuronal activityuntil the discovery of rapid eye movement (REM) sleep, its relationship with dreams and the concept of duality of sleep that emerged. When the earliest sleep EEG recordings were made, a predictable sequence of pattern was observed over the course of the night progressing from stage I sleep to stage 4. Kleitman and Dement found that this cyclical variation of EEG patterns occurred repeatedly throughout the night at intervals of 90 to 120 minutes from the end of one REM to the next REM period. The regular occurrence of REM periods and dreaming strongly suggested that dreams did not occur in response to chance disturbances. It took several years to realize that REM sleep was qualitatively different from the rest of the sleep. It was established in 1950s that electromyographic activity and muscle tone in the body was completely suppressed

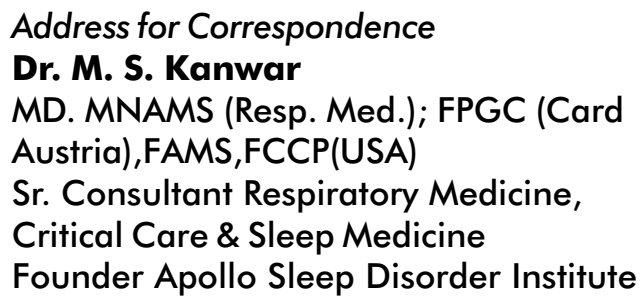

during REM periods only. Much of the initial sleep research revolved around studying dreaming and REM sleep. REM sleep was implicated in psychotic processes. Decreased REM latency was also a subject of study in endogenous depression and narcolepsy. Researchers have all along been trying to understand the formation and meaning of dreams.

In 1968 Rechtschaffen and Kales developed a standardized method of scoring the sleep recordings. The American Narcolepsy Association was formed in 1975 and in 1978 multiple sleep latency test (MSLT) was developed. Sleep labs were doing only research studies including the study of effect of benzodiazepines on sleep - until 1965 when a most dramatic finding, the discovery of sleep apnoea by many independent researchers changed the entire course of sleep medicine. And it paved the way for what was to usher in a new era in sleep medicine with widespread mushrooming of sleep laboratories which saw a transformation from a very limited research tool into a thriving clinical, diagnostic and therapeutic tool for a rapidly increasing largely a life style disease called sleep apnoea. Colin Sullivan showed in his experiments between 1980-1982 how obstructive sleep apnoea (OSA) could be abolished by giving continuous positive airway pressure (CPAP) acting as a pneumatic splint. Almost 3 decades later, CPAP till date remains the best option and easily the most widely used modality for the treatment of OSA. Before 1980s a long term tracheostomy was the only effective treatment of OSA, highly undesirable though.

A surgical approach called Uvulopalatopharyngoplasty (UPPP) became popular in 1980s particularly in Germany and France and the ENT surgeons across Atlantic also started believing that they could give permanent cure or at least dramatically reduce the severity of OSA by debulking the entire area in oropharynx and nasopharynx which was staring as an obvious visible area of obstruction on clinical examination. Perhaps it was not realized that 
pharyngeal obstruction and collapse during sleep is a dynamic process in OSA patients and that the obstruction develops at multiple levels. The fashion of UPPP gradually abated in late 1980s and early 1990s as the patients still remained symptomatic in majority except for their snoring. Perhaps those with only milder form of the disease, majorly snoring, were benefited and the rest needed CPAP. Some of them had problem of regurgitation after surgery and in some CPAP was more difficult to accept while in some the Apnoea Index was found to be even higher about 20 months after UPPP. Since the hypopharyngeal areas of obstruction and collapse could not be corrected by UPPP it dawned on everyone that the procedure would not be giving desired results except in a highly selected and milder cases of OSA. The decade of 1980s saw the publication of sleep medicine's first text book, Principles and Practice of Sleep Medicine by Kryger, Roth and Dement.

\section{The present}

In 1990s there was an acceleration in the acceptance of sleep medicine around the world but till today adequate and quality sleep medicine services are not present in most parts of the world. In Europe and North America where the awareness level of OSA has been quite high, there is excessive load on sleep labs with long waiting lists. The problem is of an entirely different nature in the developing and underdeveloped countries. Awareness levels are still low. Snoring has traditionally been linked to deep, restful and blissful sleep unless the individual is visibly struggling for breath or observed to be very restless in sleep. Several sleep labs are operational especially in the metropolitan cities in the developing countries like India. But they are not utilized to full capacity despite the fact that what we are seeing is only the tip of the iceberg. There are millions of sleep apnoeics in India but what we are diagnosing and treating are in hundreds and thousands. A disease which afflicts $2-4 \%$ of adult females and 4-9\% of adult males can not be ignored by physicians, public and the public health authorities alike given the strong links of morbidity, quality of life and mortality risks associated with it.

There is a strong data supporting the association of OSA with other life style diseases viz obesity, diabetes, hypertension and dyslipidemia, an association which of late is termed as 'Syndrome Z'. Life style diseases are the leading cause of morbidity in the developed countries and they are trying to catch up with the infectious diseases in the underdeveloped and the developing countries. Proper diagnosis and treatment of OSA as also of all the other components of 'Syndrome $Z$ ' is going to assume greater importance in future. It seems unlikely in the forseeable future that the prevalence and incidence of OSA would start to show a downward trend.

The diagnosis of sleep apnoea has traditionally depended on the fully supervised hospital based overnight polysomnography(PSG) study which is the gold standard to diagnose sleep disorders. Simpler sleep diagnostic techniques, called Level 2, 3 and 4 studies have some role to play as apnoea screen studies. They are by no means an ideal way to diagnose all aspects of even OSA but cost cutting measures have recently forced managed care organizations in USA to allow their re-imbursement. Another reason for this step could be the hope to reduce the waiting lists in the sleep labs there. Moreover, a few patients are reluctant to go in for hospital based complete PSG studies. However, the home studies too have to be scored and revalidated. A very disturbing and unethical trend is setting in some places in India. Home based sleep studies are being conducted to use AutoCPAP for titration latching on to every opportunity to sell the Auto-CPAPs without any revalidation. And who is doing it? Sales personnel without any technical certification or formal training, employed by local distributors of even well known international brands dealing in sleep labs and PAP machines. This is the most brazen, bizarre and unethical way of progression in any diagnostic and therapeutic modality in any field of medicine where a physician, let alone a sleep expert may at best be a fringe player or not in the picture at all for a disease which usually requires life long management and periodic expert counseling. This amounts to misuse of a sensitive diagnostic modality requiring high skills and training as well as application of CPAP / auto-CPAP by unqualified non-physicians or at times physicians with no training in the field whatsoever. This is akin to a non-neurologist or even a non-physician advising an MRI brain to a patient of stroke from a preferred radiology centre and then based on some sort of computerized interpretation of the MRI software, advising treatment modalities in which they have direct sales interest without the patient ever seeing a neurologist. This field can run the risk of coming into disrespute by this malpractice as well as injudicious and indiscriminate use of PAP machines especially AutoCPAP.

No wonder the lack of human touch, repeated medical counseling and scientific explaination to the patient from 
experts would be missing. The result would be poor CPAP compliance to say the least. Not only that, several individuals are put on the PAP machines when they did not need one. Only a legislation will curb this unruly practice by some. In healthcare, particularly in sleep, the core issues are human, and as such are multifaceted and multidimensional, and in order to adequately encompass those issues the combination of technical skills with the art of good clinical practice is necessary.

John Shepard showed beautifully in 1980's how OSA was linked to fluctuating blood pressure during sleep. Subsequently Shahrokh Javaheri as well as Virend Somers and several others have done some dedicated work in OSA associated with heart failure. It is good to see that cardiologists have started to appreciate the cardiovascular risk of OSA and that OSA worsens heart failure and heart failure initiates / worsens central sleep apnoea and Cheyne-Stokes breathing which in turn can worsen heart failure. Improvement with CPAP is shown in several studies. Likewise, the neurologists are also getting sensitized to the need to treat OSA and Cheyne-Stokes breathing when their patients suffer acute stroke especially to prevent any recurrent hypoxia in acute event. Furthermore, they are beginning to appreciate the positive impact on stroke prevention if OSA and OSA aggravated hypertension are treated well. Social aspects of excessive daytime somnolence, depression \& forgetfulness in gross OSA patients is being understood better by various medical specialities dealing with such patients including psychiatrists. However, we still have a long way to go in the sensitization of all physicians from various disciplines practicing far and wide. So, poor awareness of OSA in public, relatively poor sensitization among physicians, reluctance of patients to get appropriate tests done and finally reluctance in acceptance of the correct treatment are all stumbling blocks in reducing the burden of the disease in society besides the reluctance in patient efforts to keep their BMIs under control.

Sleep deprivation is becoming another important issue in sleep medicine especially in the metropolitan cities where life is very fast paced among all age groups until retirement. Social, professional and family pressures and a constant race to be an achiever amongst peers is contributing to this malady which leads to its own set of symptoms and at times chronic insomnia with anxiety and or sleep deprivation being the triggers.

Sleep medicine has come a long way in North
America, Europe and Australia with real acceleration and acceptance of sleep medicine taking place in 1990s, national sleep foundation also took shape at this time in USA. It helped in large scale research projects, develop education material and formal training programs. Year 1990 also saw the birth of American Academy of Sleep Medicine (AASM) which has accredited over 1000 sleep centres in USA. Now a full American Board of Sleep Medicine exists which regulates the formal accreditation of sleep specialists. The first text book in Sleep Medicine was published in 1989. Now you can find several books on this subject. For many years there was only one journal devoted to sleep, by 2009 there were plentiful worldwide.

In the first decade of $21^{\text {st }}$ century many researchers studied the link between the components of metabolic syndrome and OSA. Defining the precise risk of coronary heart disease (CHD) attributable to OSA is difficult because those with the latter have comorbidities such as obesity, hypertension and dyslipidemia that place many at higher risk for cardiovascular disease (CVD). Nevertheless, it has been shown that there is a stepwise increase in CVD risk factor levels with greater severity of OSA. Recently evidence has accumulated to show that patients with uncomplicated hypertension often have insulin resistance as part of metabolic syndrome. Insulin, besides its metabolic effects exerts significant actions on the sympathetic nervous system and release of nitric oxide. These activities can be involved in the short term and long term control of vascular tone, circulating volume and may affect blood pressure. Sleep deprivation contributes to the development of insulin resistance as well as other metabolic and endocrine alterations like abnormalities in cortisol, growth hormone and leptin secretion. It strongly suggests that sleep deprivation including that due to sleep disordered breathing has an adverse impact on components of metabolic syndrome and may represent a risk factor for weight gain. Resistance to appetite suppressant effects of leptin may be involved because OSA patients have higher leptin levels than similarly obese controls.

Prevalence of sleep apnoea in coronary artery disease patients has been shown to be upto two fold greater than in non-coronary artery disease subjects. Severe intermittent hypoxaemia, acidosis, increased BP, and sympathetic vasoconstriction in conjunction with simultaneous changes in intrathroacic and cardiac transmural pressures are all compelling arguments for OSA as a potential trigger for myocardial ischemia. It is

Indian Journal of Sleep Medicine (IJSM), Vol. 4, No. 4, 2009 
believed that endothelial dysfunction and systemic inflammation may promote structural coronary artery damage. Lets us also not forget that more than half of sudden cardiac deaths in patients with proven OSA occur during the sleeping hours between $10 \mathrm{pm}$ and $6 \mathrm{am}$, in contrast to those free of OSA having likelihood of sudden cardiac death between Gam and 11am. Thus we have come a long way especially in the last forty years in our understanding of sleep disorders, their pathogenesis \& pathophysiology but a lot needs to be done in furthering our understanding of a several issues discussed below. Certainly we need to come up with more and better treatment options if the society fails to keep a check on the waist lines.

\section{The future}

Recent developments have advanced our knowledge concerning relationship between OSA or sleep disordered breathing $(\mathrm{SDB})$ but there are a number of unanswered questions requiring further investigations.

- Systemic inflammation, sympathetic activation, pressure surges and oxidative stress may all contribute to the development of endothelial dysfunction. However, evidence of endothelial dysfunction in OSA patients has not been consistent. Conflicting findings with regard to resistance vessel endothelial function in OSA speak of the importance of careful exclusion of comorbidities in studies of OSA patients, though recent data suggest that treatment with CPAP may improve endothelial function.

- Studies on the treatment of OSA with CPAP have yielded inconsistent results in improvement in the metabolic disturbance. Larger trials will hopefully give us definite answers in near future.

- OSA has also been associated with increased platelet activation, increased fibrinogen and other potential markers of thrombotic risk. However, more studies are needed to more definitely evaluate the role of hemostatic mechanisms and to confirm any hypercoaguable state in OSA in larger trials. Is there a role of anti-thrombotic therapy in some patients of OSA ?.

- Pathophysiological link between sympathetic dysfunction and target organ change and structural damage in blood vessels is not well understood. Is it that the differential effect is because all blood vessels are not alike? Receptors of inflammatory mediators may be dissimilar according to the type or size of blood vessel. We will hopefully get a clearer picture on the issue.

- We need to better understand if there is a particular OSA sub-population at higher risk for CHD and if there are genetic markers that might identify these individuals.

- The future will also reveal the prevalence of OSA in those with asymptomatic heart disease. Information regarding the extent and progression of subclinical CHD in OSA might provide evidence for use of noninvasively detected biomarkers of vascular disease. Direct relationship of OSA with carotid intima-media thickness \& brachial artery flow mediated dilatation will be better understood in larger studies.

- The future will identify genes common to OSA, obesity and cardiovascular disease.

- More research is being focused on paediatric sleep. We will be wiser on several issues in coming years viz presence of a very high prevalence of central apnoea in children with Prader-Willi syndrome especially due to central adrenal insufficiency will be better studied. Larger trials will hopefully also establish that systematic management of sleep problems at or around school entry could represent a feasible way to reducing child behavior problems and improving quality of life and academic performance in these crucial early years of life.

- Oral appliances will hopefully become more acceptable, effective and more commonly used.

- Pharmaceutical therapies will emerge and assume a more important role in the treatment of milder forms of OSA.

- Patient burden will increase tremendously as awareness level increases in the Indian scenario. Hopefully, the Mediclaim/insurance will share the financial burden of diagnostics and therapeutics.

- Relationship of pregnancy, snoring and OSA will hopefully be studied in larger trials and a possible relationship with future development of metabolic syndrome established.

- OSA aggravated nocturnal angina or asymptomatic ST-T changes would hopefully be studied in larger controlled trials in CAD patients.

- A number of less intrusive and simple to operate 
diagnostic techniques are available but their validation against PSG in large number of subjects is lacking. The future will hopefully solve this problem for widespread expansion and detection of OSA in community.

- Medical therapy will hopefully bring a great relief to a lot of sleep apnoeics in future. That 'magic pill' is still awaited.

- Advances in neurobiology of sleep as well as our understanding of control of breathing during sleep will illuminate our understanding and lead to new diagnostics and therapeutics to non-respiratory disorders. The so called complex sleep apnoea will be more easily understood.

- The future should soon see a legislation or clamp down by competent medical authorities / medical councils on unethical and gross misuse of sleep laboratories and the PAP machines wherever such things are happening around the world.

- The quality control is currently poor in the Indian Sleep diagnostic \& therapeutics scenario but hopefully it should improve.

Research grows in retrograde fashion also. We see the obvious and then set out trying to prove the point. Sometimes the proof is got easily but usually the believers are outnumbered by the non-believers for whom the evidence is never hard enough. To prove a hypothesis, you have to negotiate your way through lanes and bylanes full of critics. OSA is one such example, despite a very high prevalence worldwide this disease has taken a few decades to come into the mainstream of medicine or has it? Watch it! It is beginning to happen in many parts of the world. 